\title{
First results from an uncooled LWIR polarimeter for cubesat deployment
}

\author{
Kira A. Hart, ${ }^{\text {a,* }}$ Meredith Kupinski, ${ }^{\text {a Dong Wu, }}$, and Russell A. Chipman ${ }^{\text {a }}$ \\ ${ }^{a}$ University of Arizona, Tucson, Arizona, United States \\ ${ }^{b}$ NASA Goddard Spaceflight Center, Greenbelt, Maryland, United States
}

\begin{abstract}
A compact long-wave infrared (LWIR) channeled spectro-polarimeter (IRCSP) has been developed for integration into the NASA Earth Science Technology Office (ESTO) funded submm-wave and LWIR polarimeters project to measure the microphysical properties of cloud ice. Once deployed, the IRCSP will produce the first linear Stokes measurements $\left(S_{0}, S_{1}, S_{2}\right)$ of upper-tropospheric cirrus clouds from 8.5 to $12.5 \mu \mathrm{m}$. For the first time, a compact, light-weight, and uncooled LWIR polarimeter with off-the-shelf thermal optical components is demonstrated. We report narrowband calibration measurements which quantify metrics of polarimetric system performance. The response of the system to linearly polarized light is shown to agree with both a Mueller matrix model and modulation function for narrowband calibration measurements with an $R^{2}>0.98$ from 8 to $11 \mu \mathrm{m}$. The polarimetric efficiency is $>0.8$ from 8 to $11 \mu \mathrm{m}$ for narrowband measurements indicating satisfactory performance of the polarization optics. Beyond $11 \mu \mathrm{m}$, the agreement is significantly reduced as thermal noise compounds with reduced detector response. Ultimately, the observed system performance is limited by the spectral response of the detector past $11 \mu \mathrm{m}$ in addition to the thermal noise inherent for the measurements at room temperature. (c) The Authors. Published by SPIE under a Creative Commons Attribution 4.0 Unported License. Distribution or reproduction of this work in whole or in part requires full attribution of the original publication, including its DOI. [DOI: 10.1117/1.OE.59.7.075103]
\end{abstract}

Keywords: LWIR; polarimetry; polarization; remote sensing; spectropolarimeter; cloud ice.

Paper 191639 received Nov. 25, 2019; accepted for publication Jun. 23, 2020; published online Jul. 3, 2020.

\section{Introduction}

Clouds remain a major source of uncertainty in current global climate models (GCMs). ${ }^{1}$ Ice clouds, in particular, have been used as a tuning parameter in GCMs to absorb modeling errors in the radiation budget at the top of atmosphere and precipitation at the surface. ${ }^{2,3}$ As a result, a wide spread of cloud ice values are reported from the models. ${ }^{3,4}$ The need for observational constraints on model cloud ice is highlighted in the National Research Council Earth Science Decadal Survey study (2007), in which the Aerosol-Cloud-Ecosystems Mission (ACE) recommended an advanced science payload with combined submm-wave and IR radiometers to improve the accuracy of cloud ice measurements. ${ }^{1,5}$

Remote sensing of global cloud ice has been a challenge from space. Despite great advances from CloudSat radar and other satellite sensors, accuracy of ice water path (IWP) measurements is still a factor of 2 or greater between different observations, mostly due to assumptions about ice particle size. ${ }^{3,6} \mathrm{Wu}$ and Gong have shown that an additional $40 \%$ error in microwave-derived IWP may occur for polarized radiance measurements, because cloud scattering radiances from ice particle shapes are often polarized. ${ }^{2,7}$ Scattering models have demonstrated that spectrally dependent measurement of degree of linear polarization (DOLP) would provide the needed insight into particle aspect ratio and diameter. ${ }^{4,8-10}$

The first global polarimetric study of ice crystals was performed by the Global Precipitation Measurement Microwave Imager (GMI) which investigated $\mathrm{H}-\mathrm{V}$ polarization variances in the 89- and 166-GHz channels. ${ }^{11}$ This mission attained great success demonstrating the ability to constrain particle shape factor, however uncertainty in density and size significantly impacted results. ${ }^{11}$ Higher frequency polarimetric measurements are necessary to probe crystals of smaller

*Address all correspondence to Kira Hart, E-mail: khart@optics.arizon.edu 
diameters to decode the full microphysical information. ${ }^{10,11}$ Recently, Coy et al. ${ }^{12}$ have shown that LWIR linear polarization measurements are particularly sensitive to ice crystal in optically thin clouds.

The University of Arizona in conjunction with NASA's Goddard Spaceflight Center developed a compact conical scanning LWIR polarimeter to perform the first linear Stokes measurements of cirrus clouds from 8.5 to $12.5 \mu \mathrm{m}$. As a part of the submm-wave and long-wave infrared polarimeters for cirrus ice properties (SWIRP) project, the LWIR polarimeter will operate in conjunction with two sub-mm radiometers $(220$ and $680 \mathrm{GHz})$ to measure the full dynamic range of ice particle sizes. The cloud-induced polarimetic variances at $220 \mathrm{GHz}, 680 \mathrm{GHz}$, and in the 8 to $12 \mu \mathrm{m}$ bands provide the wide dynamic range in sensitivity needed for measuring IWP $>5 \mathrm{~g} / \mathrm{m}^{2}$ and particle diameters $<30 \mu \mathrm{m}^{2,13}$ The science requirement of at least $1 \mathrm{~K}$ thermal resolution in this waveband enables the measurement of bulk particle shape for diameters below $30 \mu \mathrm{m} .{ }^{2}$ Once assembled, the full SWIRP instrument will have a volume of $<11 \mathrm{~L}$ and is viable for both CubeSat or small distributed flight system deployment.

An infrared channeled spectropolarimeter (IRCSP) approach was chosen to achieve full linear Stokes measurements in a compact housing without the need of moving components. ${ }^{14}$ With a volume of $120 \mathrm{ml}$, the polarimeter is designed to be compact, cost-effective, and low risk while maintaining high performance. The IRCSP performs a spectrally dependent polarization modulation to produce a cosinusoidal intensity pattern at the detector whose intensity is proportional to DOLP. The angle and amplitude of the intensity patterns produced by the IRCSP are used to retrieve the angle of linear polarization (AOLP) and DOLP.

Channeled-polarimeters have been shown to be effective in producing instantaneous hyperspectral polarimetric data without sacrificing spatial resolution and have many remote sensing applications. ${ }^{15-22}$ Iannarilli et al. previously demonstrated an LWIR polarimetric hyperspectral imager capable of measuring the DOLP and AOLP of light scattered from ocean surfaces to estimate local surface orientation. ${ }^{19}$ Channeled spectropolarimeters have also been applied to ground mine detection and atmospheric aerosols sensing from space. ${ }^{23,24}$ In addition, the SPEX-airborne hyperspectral multiangle polarimeter demonstrated success utilizing a channeled polarimeter for aerosol characterization in the visible. ${ }^{16-18,22,25,26}$ Due to ozone-induced atmospheric attenuation in the thermal IR, it is necessary to measure both orthogonal modulated paths in order to perform both radiometric and polarimetric measurements across the spectrum. van Harten et al. ${ }^{25}$ previously demonstrated success using a dual-path approach to maintain accuracy in spectral line polarimetry.

The final SWIRP IRCSP instrument has a total dimension of $2 \times 2 \times 10 \mathrm{~cm}$ and a steady state power consumption of $0.5 \mathrm{~W}$. To achieve this compact and efficient specification, the IRCSP is constructed using a combination of birefringent crystals serving as multiorder waveplates, and a wiregrid linear polarizer (LP) tiled at 20 deg to modulate the signal in both transmission and reflection. While single-detector solutions such as Wollaston prisms and polarization gratings were considered, a dual-camera LP approach was chosen to demonstrate the instrument on schedule and reduce risk. In the dual-camera design, the LP is followed by two identical optical paths consisting of diffraction gratings (DG) and uncooled microbolometer detectors. The compact and efficient design is possible due to the recent availability of low-noise equivalent differential temperature and compact uncooled microbolometers. Long-term deployment and radiometric calibration of these detectors been successfully demonstrated by Nugent and Shaw. ${ }^{27}$ In addition, Polaris Sensor Systems has shown success utilizing these uncooled detectors for LWIR linear imaging Stokes camera by applying a microfilter array close to the microbolometer focal plane. ${ }^{28,29}$

\section{Theory}

\subsection{Linear Stokes Polarimetry}

The Stokes parameters uniquely quantify the polarization state of incoherent light with four numbers: $\left[S_{0}, S_{1}, S_{2}, S_{3}\right]$. The first component, $S_{0}$, is the total radiance, and the other components also have units of radiance. The normalized unitless Stokes parameters $\mathbf{S}=\mathbf{S} / S_{0}=\left[1, s_{1}, s_{2}, s_{3}\right]$ 
are similarly represented by lower case $\mathbf{s}$. The definitions of linear Stokes parameters $S_{1}$ and $S_{2}$ are associated with a given coordinate system. Circular polarization $S_{3}$ is typically negligible for sunlight scattered by the atmosphere or Earth's surface. The linear Stokes components are often combined into a dimensionless quantity, the DOLP

$$
\mathrm{DOLP}=\rho=\frac{\sqrt{S_{1}^{2}+S_{2}^{2}}}{S_{0}}=\sqrt{s_{1}^{2}+s_{2}^{2}}=\frac{I_{\mathrm{pol}}}{A},
$$

where $I_{\mathrm{pol}}$ is the polarized radiance (the fraction of light in the scene that is polarized) and $A=$ $S_{0}$ is the total radiance. ${ }^{30}$ The AOLP is

$$
\mathrm{AOLP}=\theta=\frac{1}{2} \tan ^{-1}\left(\frac{s_{2}}{s_{1}}\right)
$$

Note that DOLP is invariant to the coordinate system but AOLP depends on the coordinate system. A $3 \times 1$ linear Stokes vector is expressed in terms of $A, \rho$, and $\theta$ as

$$
\mathbf{S}(\lambda)=A_{\lambda}\left[1, \rho_{\lambda} \cos 2 \theta_{\lambda}, \rho_{\lambda} \sin 2 \theta_{\lambda}\right]^{t}
$$

where $t$ denotes transpose ${ }^{30}$ Here, $A_{\lambda}$ is the spectral intensity of the target and both AOLP: $\theta_{\lambda}$ and DOLP: $\rho_{\lambda}$ are functions of wavelength. The notations for Stokes parameters in this document are $\mathbf{S}$ is the polarization state of the object, $\tilde{\mathbf{S}}$ is the polarization state incident on the camera, and $\hat{\mathbf{S}}$ is the reconstructed linear Stokes vector. In a noise-free model $\tilde{\mathbf{S}}=\mathbf{M S}$ where $\mathbf{M}$ is the cumulative Mueller matrix of the optical system.

\subsection{Dual-Path Channeled Spectropolarimeter}

A channeled spectropolarimeter is utilized to measure the spectrally dependent AOLP and DOLP in a compact instrument. This instrument is comprised of a series of polarization modulation components followed by a DG and imaging lens, which image a polarization modulated spectrum at the focal plane. The polarization modulation is performed by a quarter-wave retarder (QWR) with a fast-axis at $45 \mathrm{deg}$ followed by a CdSe crystal high-order retarder (HOR). This combination acts as a wavelength-dependent circular retarder where the magnitude of the retardance is inversely proportional to wavelength. The thickness and birefringence of the HOR were selected to produce a difference of four waves of retardance from 8.5 to $12.5 \mu \mathrm{m}$.

In the SWIRP instrument, the rotated polarized light is modulated by a wiregrid LP tilted at $20 \mathrm{deg}$, which separates the 0-deg and 90-deg polarization states as a polarizing beam splitter. The reflected and transmitted paths are both measured by two different cameras to distinguish wavelength-dependent transmission from polarization-dependent transmission. ${ }^{14}$

For unpolarized light, the flux is evenly divided into reflected and transmitted paths by the LP since the QWR and HOR do not induce polarization. When linearly polarized light is incident, the output polarization from the QWR and HOR is rotated as a function of the wavelength, as shown in Fig. 1. The relative transmission and reflection from the LP then depend on wavelength. This transmission and reflection through the linear polarization are periodic functions with opposite polarities. The amplitude of modulation is proportional to the DOLP, and the phase offset is proportional to AOLP. The SWIRP instrument is designed to measure DOLP and AOLP with $1-\mu \mathrm{m}$ resolution, as rapid variation with wavelength is not expected. ${ }^{12}$ Finally, the DG and imaging lens form a spectrum at the focal plane forming a $64 \times 2$ array for each polarization channel, 64 for the spectral sampling, and 2 for spatial sampling. The amplitude and phase of the spectral modulation in each $1-\mu \mathrm{m}$ band provide the polarization measurement. 


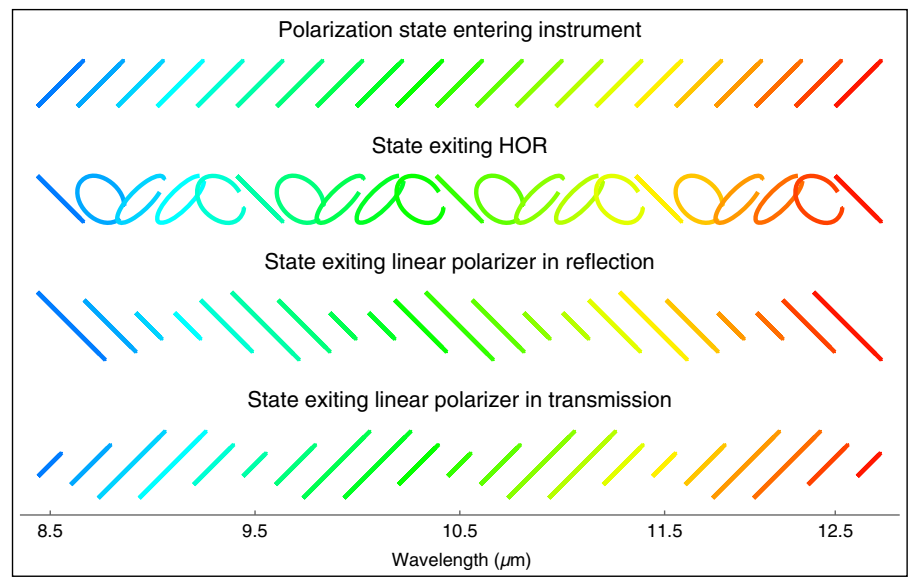

Fig. 1 The transformation of broadband linearly polarized light with constant DOLP and AOLP as propagated through the IRCSP Mueller matrix model. The polarization is altered as a function of wavelength as it passes through the QWR and HOR. Following the HOR, the beam is split and modulated by a linear polarizer in reflection and transmission.

\subsection{Dual-Path Mueller Matrix Model}

The Mueller matrix model for the two paths to each camera $c=1,2$ is

$$
\begin{aligned}
& \mathbf{M}_{c}(\theta, \lambda)=\gamma_{\mathrm{FPA}}^{c}(\lambda, \theta) \gamma_{\mathrm{DG}}^{c}(\lambda, \theta) \mathbf{P}^{c}(\lambda), \\
& T_{\mathrm{HOR}}(\lambda, \theta) \mathbf{L R}[\delta(\lambda), 0 \operatorname{deg}] T_{\mathrm{QWP}}(\lambda, \theta) \mathbf{L R}[\pi / 2+\eta(\lambda), 45 \operatorname{deg}] T_{\mathrm{Lens}}(\lambda, \theta) .
\end{aligned}
$$

Here bold uppercase letters denote $4 \times 4$ Mueller matrices, $T$ are scalar-valued transmissions, and $\gamma$ are scalar-valued efficiency. The spectral absorptivity of the focal plane array (FPA) and efficiency of DG are denoted $\gamma_{\mathrm{FPA}}^{c}$ and $\gamma_{\mathrm{DG}}^{c}$, respectively. The transmission of the lens, the HOR, and the QWR are $T_{\mathrm{Lens}}, T_{\mathrm{HOR}}$, and $T_{\mathrm{QWP}}$, respectively. The dependence of these values on wavelength and AOLP is denoted by the arguments $(\lambda, \theta)$. The two arguments of the linear retarders (LR) are the magnitude of retardance followed by the orientation of the fast-axis. The term $\mathbf{L R}[\delta(\lambda), 0 \mathrm{deg}]$ is a horizontal fast-axis linear retarder with a wavelength-dependent retardance. The term $\mathbf{L R}[\pi / 2+\eta(\lambda), 45 \mathrm{deg}]$ is a quarter-wave linear retarder oriented at $45 \mathrm{deg} .{ }^{30}$ The departure from an ideal quarter-wave of retardance is modeled by the wavelength-dependent parameter $\eta(\lambda)$. The linear polarizer is represented as $\mathbf{P}^{c}$ where the superscript denotes the reflected or transmitted path to the camera $c$. To simplify Eq. (4), the scalar transmission and efficiency terms are combined. Components upstream of the linear polarizer are $\alpha(\lambda, \theta)=T_{\text {Lens }} T_{\mathrm{HOR}} T_{\mathrm{QWP}}$. After the linear polarizer, the polarization in each path will be constant $\beta_{c}\left(\lambda, \theta=\theta_{c}\right)=\beta_{c}(\lambda)=\gamma_{\mathrm{FPA}}^{c} \gamma_{\mathrm{DG}}^{c}$.

The wavelength-dependent intensity measured by camera $c$ is related to the cumulative system Mueller matrix $\mathbf{M}_{c}$ and the polarization state of the object $\mathbf{S}$ as

$$
\tilde{I}_{c}(\rho, \theta, \lambda)=\left[\tilde{\mathbf{S}}_{c}(\rho, \theta, \lambda)\right]_{0}=\left[\mathbf{M}_{c}(\theta, \lambda) \mathbf{S}(\rho, \theta, \lambda)\right]_{0},
$$

where $[.]_{0}$ denotes the first element, i.e., intensity $\tilde{I}_{c}$, of the Stokes vector $\tilde{\mathbf{S}}_{c}$ incident on camera $c$. Simplifying the matrix-vector product on the RHS of Eq. (5) results in an intensity pattern that is periodic in wavelength

$$
\tilde{I}_{c}(\rho, \theta, \lambda)=\frac{1}{2} A_{\lambda} \alpha(\lambda, \theta) \beta_{c}(\lambda)\left\{\Sigma_{c}(\lambda) \pm \Delta_{c}(\lambda) \rho_{\lambda}\left[\epsilon(\lambda) \cos 2 \theta_{\lambda} \sin \delta(\lambda)+\cos \delta(\lambda) \sin 2 \theta_{\lambda}\right]\right\},
$$

where the \pm is addition for the transmitted $c=1$ path and subtraction for reflection $c=2$. Wavelength dependence is denoted as an argument to a function for the optical components and as subscripts to the AoLP, $\theta_{\lambda}$, and DoLP, $\rho_{\lambda}$, of the incident Stokes vector. The spectral 
dependence deviation from the ideal quarter waveplate retardance is $\epsilon(\lambda)=\cos [\eta(\lambda)]$. The maximum transmission and reflection of the linear polarizer are denoted $T^{\max }, T^{\min }, R^{\max }$, and $R^{\min }$. These are combined $\Sigma_{1}=T^{\max }+T^{\min }, \quad \Delta_{1}=T^{\max }-T^{\min }, \quad \Sigma_{2}=R^{\max }+R^{\min }, \quad$ and $\Delta_{2}=R^{\max }-R^{\min }$. In total, nine parameters: $\alpha(\lambda, \theta), \beta_{c}(\lambda), \Sigma_{c}(\lambda), \Delta_{c}(\lambda), \epsilon(\lambda), \delta(\lambda)$ are fit to calibration measurements at varying incident AOLPs and wavelengths. In an ideal system: $T^{\min }=0, T^{\max }=1, \eta=0$, and $\alpha(\lambda, \theta)=\beta^{i}(\lambda)=1 ; \forall \lambda$. Thus for the ideal system the fringe pattern reduces to

$$
\tilde{I}_{c}(\rho, \theta, \lambda)=\frac{A_{\lambda}}{2}\left\{1 \pm \rho_{\lambda} \sin \left[\delta(\lambda)-2 \theta_{\lambda}\right]\right\}
$$

where the spectrally dependent retardance $\rho_{\lambda}$ defines the frequency of a sinusoidal pattern; this is called the carrier frequency. The magnitude of this sinusoidal pattern is the DOLP $\rho_{\lambda}$ and the phase shift is proportional to AOLP $\theta_{\lambda}$.

\subsection{Modulation Function}

The Mueller matrix model described in the previous section provides a continuous representation of all polarization-dependent and polarization-independent transmission, efficiency and retardance of the system. An additional performance characteristic in spectro-channeled polarimetry is the spectral blurring due to the finesse of the DG and the field stop geometry. Consider the onedimensional spatial distribution of a monochromatic source at the FPA denoted by $f_{c}\left(\lambda_{l}, x\right)$. An interplay of the DG's finesse and the field stop geometry determine the overlap of this distribution for adjacent wavelengths. ${ }^{31}$ In a pixel-sampled continuous to discrete model, the monochromatic intensity given by the Mueller matrix model in Eq. (6) can be expressed instead as a set of broadband noise-free measurements

$$
P_{c, j}(\rho, \theta)=\sum_{l=0}^{L} \tilde{I}_{c}\left(\rho, \theta, \lambda_{l}\right) \int_{x_{j}}^{x_{j+1}} f_{c}\left(\lambda_{l}, x\right) \mathrm{d} x
$$

where $P_{c, j}$ is a noise-free measurement from camera $c$ and pixel $j$. Each noise-free measurement is an incoherent sum of $L$ wavelengths $\lambda_{l}$ that contribute to pixel $j$ where the pixel has an active area pitch of $x_{j+1}-x_{j}$. In Eq. (8), the modulation of $\tilde{I}_{c}\left(\rho, \theta, \lambda_{l}\right)$ is effectively blurred by broadband incident light.

To estimate an intensity $\hat{I}_{c}\left(\lambda_{l}\right)$ at wavelength samples $\lambda_{l}$, a spatial average is computed over the range of $f_{c}\left(\lambda_{l}, x\right)$. Narrowband calibration measurements are used to estimate $f_{c}\left(\lambda_{l}, x\right)$.

To characterize spectral blurring, the SPEX polarimeter ${ }^{16,25}$ defined a modulation function

$$
M\left(\lambda_{l}\right)=\frac{\hat{I}_{1}\left(\lambda_{l}\right)-\hat{I}_{2}\left(\lambda_{l}\right)}{\hat{I}_{1}\left(\lambda_{l}\right)+\hat{I}_{2}\left(\lambda_{l}\right)}=W\left(\lambda_{l}\right)\left\{S_{1} \cos \left[\frac{2 \pi \delta\left(\lambda_{l}\right)}{\lambda_{l}}\right]-S_{2} \sin \left[\frac{2 \pi \delta\left(\lambda_{l}\right)}{\lambda_{l}}\right]\right\}
$$

where $W(\lambda)$ is termed the polarimetric efficiency, and $\delta(\lambda)$ is the carrier frequency in Eq. (6). These two parameters are fit to calibration data. All linear terms in Eq. (6) are combined into the polarimetric efficiency. A noise-free simulation of $\hat{I}_{c}$ and associated modulation function are shown in Fig. 2. The phase of $M(\lambda)$ depends on the AOLP, and the amplitude depends on both the polarimetric efficiency and DOLP. Due to dispersion, the frequency of modulation is higher at lower wavelengths.

\section{Optical Design}

\subsection{Dual-Path Optical Design}

In the preliminary design, the linear polarizer was not tilted and only the modulated transmitted intensity was measured. Once the Mueller matrix data reduction was modeled using atmospheric and system transmission data, it became apparent that the ozone absorption band between 9.5 


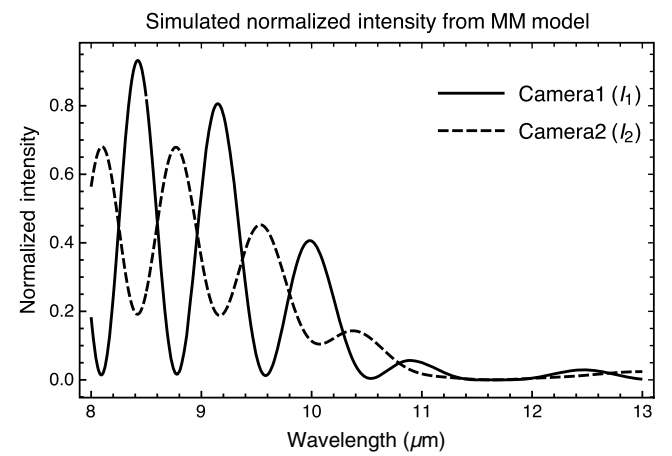

(a) Normalized intensity

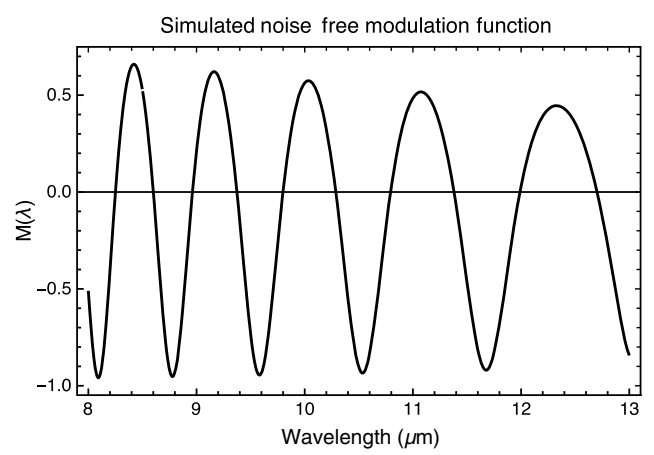

(b) Modulation function

Fig. 2 Simulated noise-free modulation function: (a) the intensity in paths 1 and 2 and (b) the modulation function. The frequency of modulation is proportional to $1 / \lambda$ due to the dispersion of $\mathrm{CdSe}$. Reduced contrast from the linear polarizer in reflection causes the modulation function to be reduced.

and $10.5 \mu \mathrm{m}$ would produce a significant artifact to the order of 1 modulation period, reducing the accuracy of measurements from 9 to $11 \mu \mathrm{m}$. Since the ozone band extends over one period of modulation, both 0 -deg and 90-deg polarization outputs of the modulation elements must be preserved to distinguish between source radiance and degree of polarization.

There are several drawbacks to the dual-path approach. First, a single camera configuration is ideal to preserve power and reduce noise. Second, the most significant departure in SWIRP from the ideal model in Eq. (7) is due to the linear polarizer's varied performance in reflection and transmission. The closest realization of the ideal system would be achieved using a Wollaston prism to image both modulated paths with high contrast onto the same detector. However, due to the risk associated with obtaining and then evaluating a CdSe Wollaston prism or an LWIR polarization grating, the linear polarizer approach was chosen to demonstrate the instrument concept.

\subsection{Microbolometer Selection}

In the first instrument prototype, FLIR's Tau2 was chosen due to its demonstrated performance. ${ }^{27}$ However, due to the tight spatial requirements imposed by other subassemblies inside the full SWIRP instrument, even a single Tau2 camera could not be oriented to fit in the drum. For this reason, FLIR's Boson uncooled microbolometer was chosen to replace the Tau2. While the Boson's $2.1 \times 2.1 \times 1 \mathrm{~cm}$ footprint met the form factor requirements, the detector has not been previously demonstrated in a spectral imaging application, nor was spectral response information for the camera available at the time of purchase. Extensive characterization of this detector's performance and stability over time will be the subject of ongoing work for this instrument and is likely be the ultimate limiting factor in the instrument's performance. However if successfully stabilized, the Boson's low-cost, low power, light-weight, and compact nature make it attractive for the use in rapidly deployable LWIR remote sensing.

\subsection{Prepolarization Optics}

The front aperture of the system consists of two collimating lenses with a field stop. This assembly serves to reduce stray light in the system, reduce systemic polarization introduced by the Fresnel coefficients for rays with large AOIs, and match the spatial resolution the IRCSP to that of the sub-mm instruments. The field stop dimension perpendicular to the plane of diffraction from the gratings dictates the instruments spatial field of view, and the dimension parallel to the field of view dictates the instrument throughput and spectral resolution. The tradeoff between throughput and spectral resolution and the optimization of field stop dimension is the subject of ongoing work. For this preliminary characterization, a $100-\mu \mathrm{m}$ field-stop was chosen to maximize throughput. For a field-stop of this dimension, cumulative diattenuation 


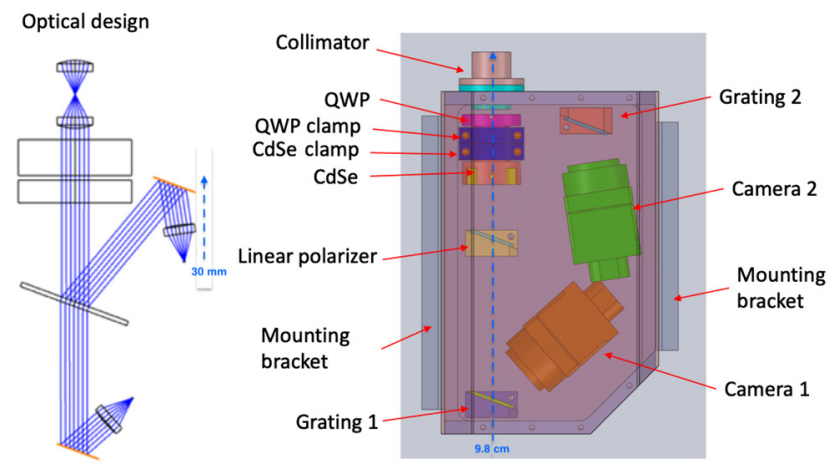

(a) Dual path IRCSP optical layout

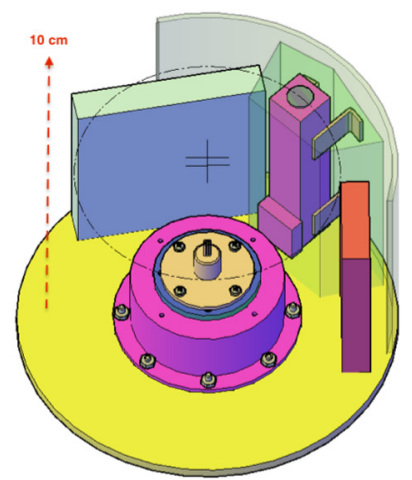

(b) Internal drum view

Fig. 3 (a) Optical layout of dual-path IRCSP and (b) inside of the instrument with the lid removed. The maximum allowable envelope for the IRCSP subsystem is $120 \mathrm{ml}$ or $0.12 \mathrm{U}$ and is shown as the transparent green box in (b). This volume constraint for the IRCSP limited the detector choice to uncooled microbolometers.

is estimated to be $<0.005$ across the entrance pupil. For the analysis presented in this work, we assume the effect of polarization-dependent transmission to be negligible compared to the reduction in polarimetric efficiency from spectral blurring and linear polarizer contrast.

\subsection{Polarization Optics}

The choice of optical elements was driven by performance, availability, and form-factor requirements. CdSe was selected as the birefringent material for the HOR due to its thermal stability and well-documented performance in the LWIR. An off the shelf cadmium thiogallate (CdGa2S4) zero-order wave-plate from Edmund Optics was purchased as the QWR. The HOR was designed to produce four waves of variation in retardance across 8.5 to $12.5 \mu \mathrm{m}$. Using Polaris-M polarization raytracing software from Airy Optics, the required thickness was calculated to be $5.01 \mathrm{~mm} \cdot{ }^{32-34}$ In general, the net retardance of any birefringent crystal will vary with temperature as the material expands or contracts. ${ }^{35}$ To calculate the net retardance as a function of wavelength and temperature, the linear coefficient of thermal expansion is combined with the Sellmeier coefficients for CdSe as described by Hale and Day. ${ }^{35,36}$ For the IRCSP HOR, the deviation in net retardance across the bandwidth is calculated to be less than one-fifth of a wave for temperatures of $270 \pm 30 \mathrm{~K}$. While the retardance of the QWR is known to be $<\lambda / 40$ at $20^{\circ} \mathrm{C}$, the measurements of the retardance at lower temperatures are not available. However, $\mathrm{CdGa}_{2} \mathrm{~S}_{4}$ has demonstrated thermal stability and hardness for temperatures $<500^{\circ} \mathrm{C}$, and thermal retardance variation is assumed to be minimal for this zero-order retarder. ${ }^{37}$

\subsection{System Assembly}

The system was assembled in the Large Optic Fabrication and Testing Facility at the University of Arizona in June 2019. With the exception of the CdSe crystal, all components can be obtained either off the shelf or with minimal lead times and included: a Moxtek wiregrid polarizer, BD-2 collimating lenses from Thorlabs, and a ruled blazed grating from OPCO. The instrument's mechanical housings are all anodized aluminum, and an aperture mount was produced to enable future integration into the full SWIRP drum as seen in Fig. 3. Assembled, the full system has a volume of $120 \mathrm{ml}$ and is approximately the size of a smart phone.

\section{Methods}

To characterize the assembled IRCSP, the response of the system as a function of AOLP and wavelength for fully and unpolarized light is measured. 


\subsection{Data Pipeline}

The two FLIR Boson cameras are configured using the software developers kit (SDK) and the Video Power and Communication Interface Module produced by OEM Cameras to simultaneously collect measurements using master-slave mode. The data acquisition software is configured such that all auto-gain and preprocessing look-up tables are disabled to return a raw 16-bit count for the $M=90 \times N$ region of interest $R_{c}[m, n]$.

For this demonstration, data were collected using the python camera control software developed using the SDK by the University of Arizona. The development of the on-board data handling and power regulation for the IRCSP to meet power and data rate requirements for the whole SWIRP assembly is ongoing at GSFC.

\subsubsection{FPA temperature nonuniformity correction}

The first step in the data pipeline is to correct for the change is response due to any variation in focal plane temperature. Utilizing the calibration algorithm developed by Nugent and Shaw, the corrected signal is given

$$
C_{c}[m, n]=\frac{R_{c}[m, n]-b[m, n](\Delta T)}{1-m[m, n](\Delta T)},
$$

where $b$ and $m$ are functions of the difference between the cameras' current FPA temperature and a reference temperature $\Delta T .^{27}$ The calibration coefficients have been previously determined at each pixel by measuring the variation is response as a function of FPA temperature. ${ }^{14,27}$

\subsubsection{Dark-field correction}

After the NUC, a dark-field correction is applied to reduce the effects of stray thermal radiation within the instrument. In flight, the dark-field images will be collected once per drum revolution via a space view. For in lab calibration, the dark-field images are recorded once every wavelength scan (100 images) with the optics blinded. The dark-field images are then corrected for FPA temperature using the NUC correction described in Sec. 4.1.1. The dark-field corrected image is then calculated

$$
C_{c, \mathrm{corr}}[m, n]=C_{c}[m, n]-D_{c}[m, n]
$$

where $D_{c}[m, n]$ is the dark-field image for camera $c$.

\subsubsection{Spectral assignment}

The final step in the preprocessing data pipeline is to associate the pixel values with wavelength. During calibration, a monochromator is utilized to determine the pixel location of the peak response at each wavelength. In order to locate the signal at wavelengths where the signalto-noise ratio (SNR) is reduced, the pixel location is fit to a linear model as seen in Fig. 4. The intensity at a given wavelength is then calculated as the average of the pixels adjacent to location of maximum response. The number of averaged pixels is dependent on the physical size of the diffracted order. For the $100-\mu \mathrm{m}$ pinhole installed in this configuration and a source bandwidth of $94 \mathrm{~nm}$, the size of the diffracted order is 6 pixels, and 3 pixels are averaged to estimate the intensity at a given wavelength. For this publication, intensity units are reported as the corrected analog digital count.

\subsection{Model Fit}

To characterize the instrument performance, the response of the system as a function of AOLP is fit to both the Mueller matrix model in Eq. (6) and the modulation function in Eq. (9) at each wavelength. The coefficient of determination $R^{2}$ describes how well the data are explained by the model for the given input parameters 


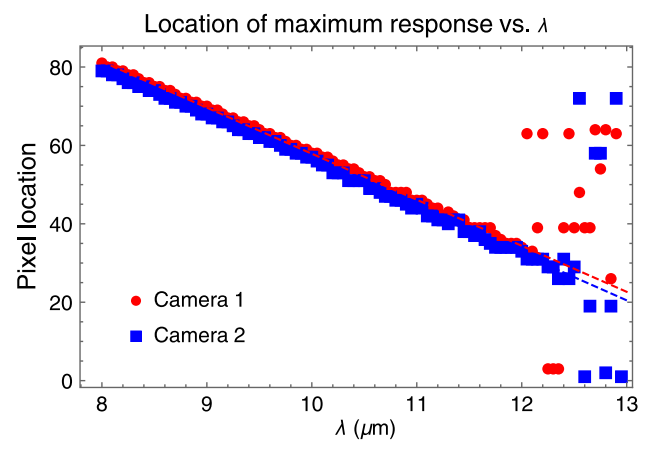

Fig. 4 The pixel location of the diffracted order in each path as a function of wavelength. A linear fit is applied to the pixel location of the maximum intensity calculated during calibration.

$$
R^{2}=1-\frac{\sum_{i=1}^{N}\left(y_{i}-\hat{y}\right)^{2}}{\sum_{i=1}^{N}\left(y_{i}-\bar{y}\right)^{2}}
$$

where $y$ are the data, $\hat{y}$ is the model estimate, and $\bar{y}$ is the sample mean of the data for a given input. This value can be used to indicate how well the model describes the data, where $R^{2}=1$ indicates an ideal fit and $R^{2}=0$ indicates no correlation between the data and model. The $R^{2}$ values for the Mueller Matrix model and modulation function are reported as a metric of their ability to describe the measured instrument performance.

\section{Results}

In June 2019, the IRCSP was delivered to NASA Goddard Space Flight center for first light characterization. To evaluate the response of the system as a function of wavelength, the system was placed in a testbed with a monochromator and blackbody source at $1000^{\circ} \mathrm{C}$. All tests were performed at room temperature and 5-five independent measurements were taken in each configuration. For all measurements the monochromator was operated with a slit size corresponding to a spectral resolution of $94 \mathrm{~nm}$. The output of the monochromator can be assumed to be unpolarized.

\subsection{System Response}

As anticipated and shown in Fig. 6, the unpolarized response of the system in reflection and transmission is not identical. Specifically, the transmitted path in camera 1 observes a sharper

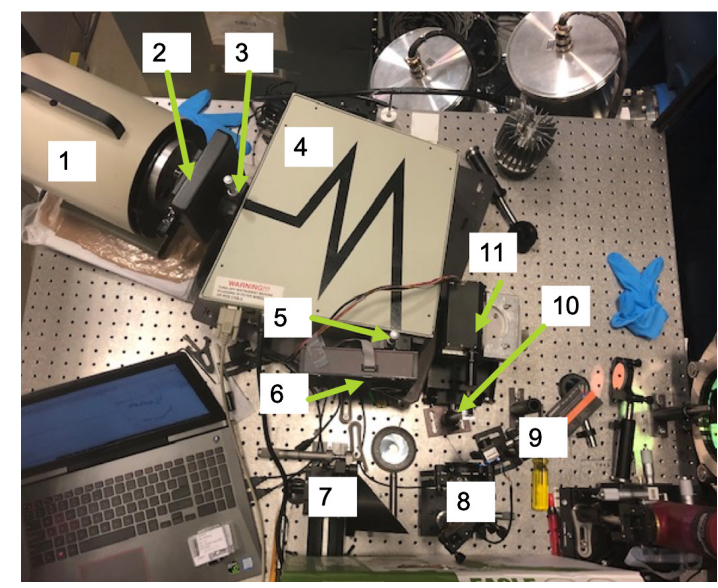

Fig. 5 Layout of the monochromator testbed at GSFC. The components are 1. black body source, 2. optical chopper, 3. monochromator input slit, 4. monochromator, 5. output slit, 6 . spectral filter wheel, 7. off-axis parabolic mirror, 8. fold mirror, 9. reference detector, 10. linear polarizer on rotating mount, and 11. IRCSP. 
decrease in efficiency at $9 \mu \mathrm{m}$ than in reflection. The response in both cameras is nearly identical past $10 \mu \mathrm{m}$ where the responsively of the detector is the dominant factor. These variations are likely due to the difference in grating efficiency for the orthogonal polarization states exiting the linear polarizer. The signal-to-noise ratio (SNR) is plotted in Fig. 9. At room temperature, the system achieves SNR $>100$ from 8.5 to $10.5 \mu \mathrm{m}$. The SNR are similar for each camera.

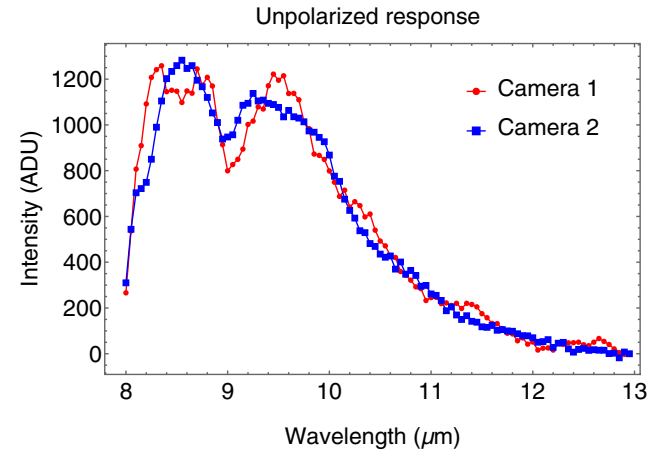

(a) Unpolarized response

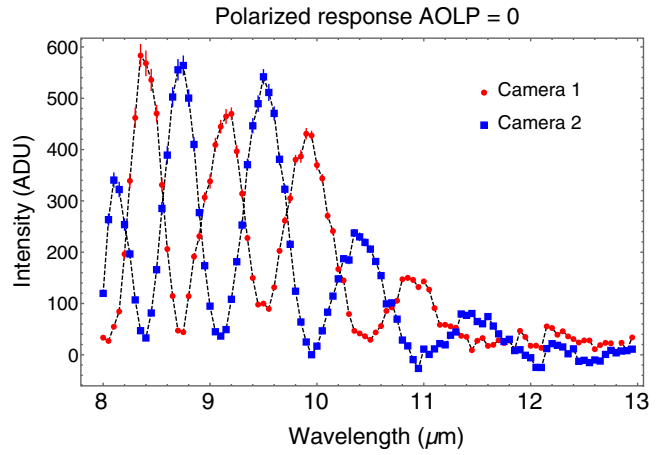

(b) Polarized response

Fig. 6 Measured response $\hat{l}_{c}$ in camera 1 (reflection, red) and camera 2 (transmission, blue) to: (a) unpolarized light and (b) polarized light with DOLP $=1$ and AOLP $=0$ deg. The response is reported as the analog digital count (ADU). Departure from equal cameras 1 and 2 measurements for unpolarized illumination indicates polarization-dependent efficiency in the DG following the linear polarizer.

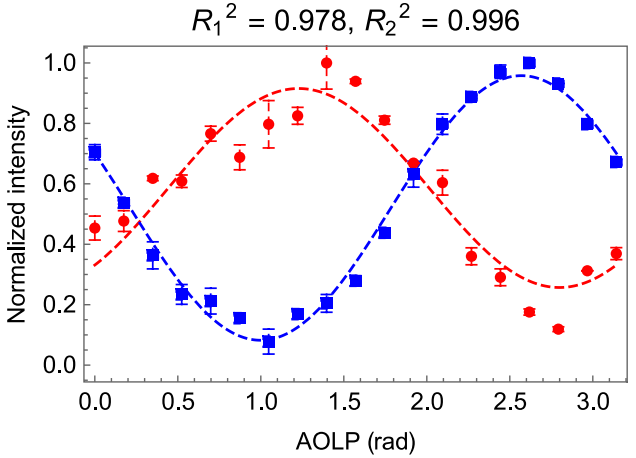

(a) $\lambda=8.5 \mu \mathrm{m}$

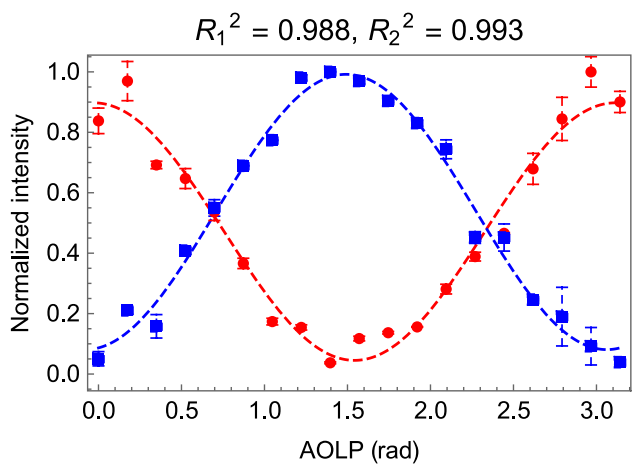

(c) $\lambda=10.5 \mu \mathrm{m}$

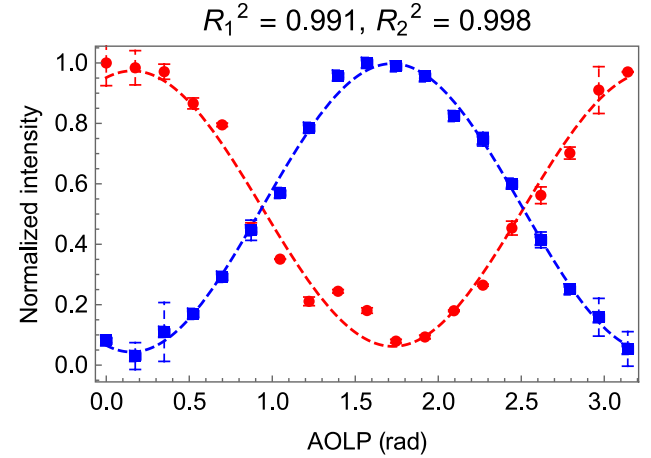

(b) $\lambda=9.5 \mu \mathrm{m}$

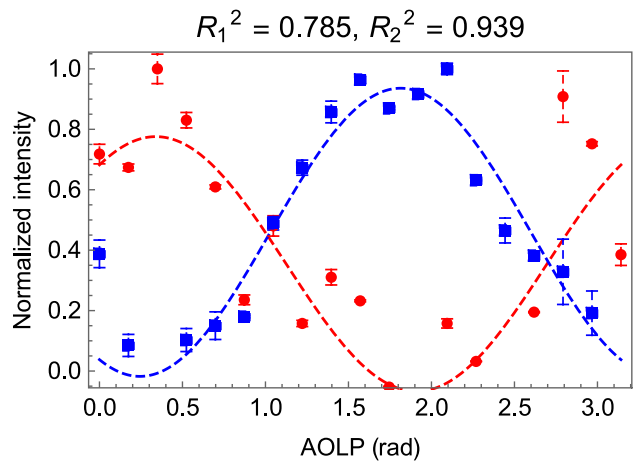

(d) $\lambda=11.5 \mu \mathrm{m}$

Fig. 7 Fit of normalized intensity in camera 1 (red) and camera 2 (blue) to Mueller matrix model in Eq. (6) for $\lambda=8.5,9.5,10.5$, and $11.5 \mu \mathrm{m}$. $R^{2}$ [Eq. (12)] values for each fit are shown above figures. Modulation is better in the transmitted path, as seen by the higher camera $2 R^{2}$ as compared to camera 1. A significant reduction in $R^{2}$ values for both cameras is observed at the highest wavelength. 
To test the polarization-dependent response of the system, a rotating linear polarizer was placed between the monochromator output and the instrument's front aperture. The tabletop setup with the linear polarizer is shown in Fig. 5. The linear polarizer was rotated to produce measurement with DOLP $=1$ and AOLP $\in[0 \mathrm{deg}, 180 \mathrm{deg}]$ for 100 wavelengths between 8 and $13 \mu \mathrm{m}$. The average response of the instrument at each wavelength for AOLP $=0 \mathrm{deg}$ is shown in Fig. 6(b). Figure 6(b) shows the expected modulation with opposite polarity in each path.

\subsection{Monochromator Calibration}

To evaluate the systemic polarization error, we first evaluate the fit of the data taken using the monochromator to both the Mueller matrix model in Eq. (6) and the modulation function in Eq. (9). As a result, spectral blurring is minimized and the polarimetric performance of the system is sampled with a narrowband illumination of $94 \mathrm{~nm}$ spectral bandwidth.

\subsubsection{Fit to Mueller matrix model}

For the Mueller matrix model fit, the polarization-independent transmission parameter $\alpha$ from Eq. (6) is first fit to the unpolarized response in Fig. 6 and used to normalize the response of the polarized measurements (Fig. 8). The normalized intensity of the system at each pixel as a function of AOLP is then fit to the eight additional parameters from Eq. (6). The result of this fit and the associated $R^{2}$ values are shown in Fig. 7 for 8.5, 9.5, 10.5, and $11.5 \mu \mathrm{m}$. As expected from the results in Fig. $9, R^{2}$ is reduced past $11 \mu \mathrm{m}$. For all wavelengths, the fit in camera 2 outperforms that for camera 1.
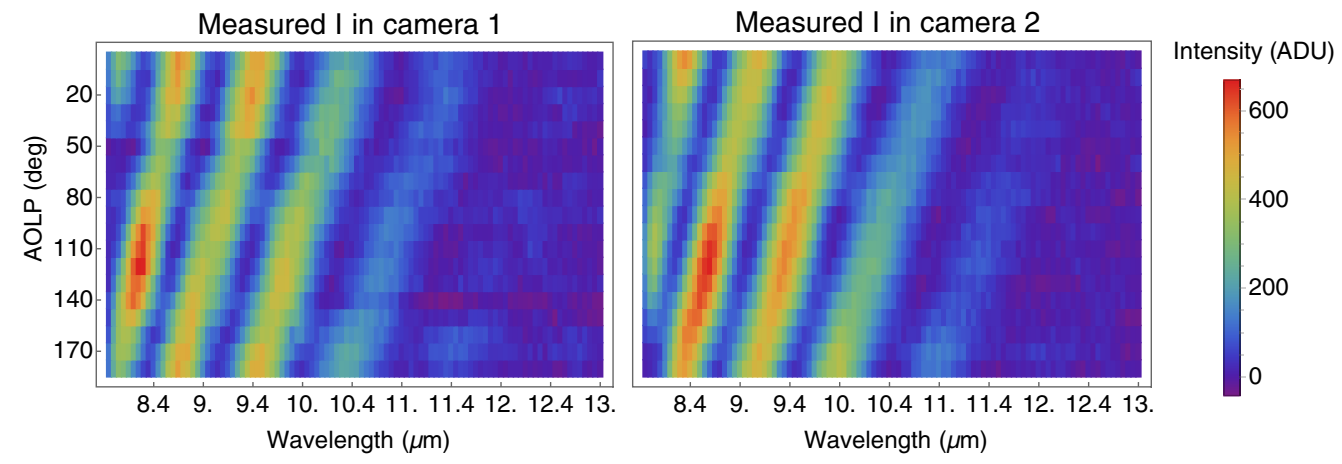

Fig. 8 The estimated intensity $\hat{l}_{c}$ as a function of AOLP and wavelength for DOLP $=1$ calculated using the spectral assignment procedure discussed in Sec. 4.1.3.

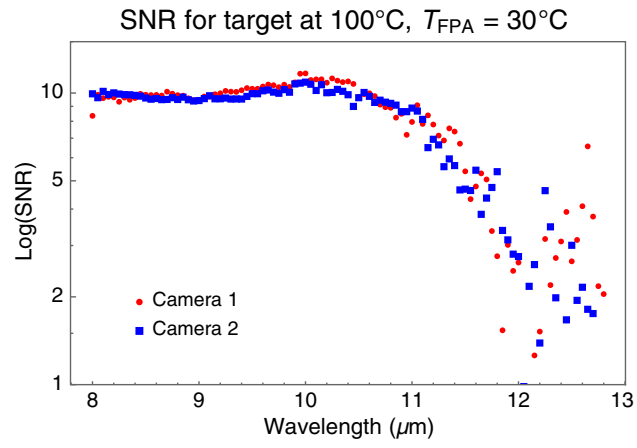

Fig. 9 The calculated log (SNR) at room temperature for cameras in path 1 (reflection) and path 2 (transmission) to unpolarized light calculated from the data in Fig. 6. SNR declines abruptly past $11 \mu \mathrm{m}$. 


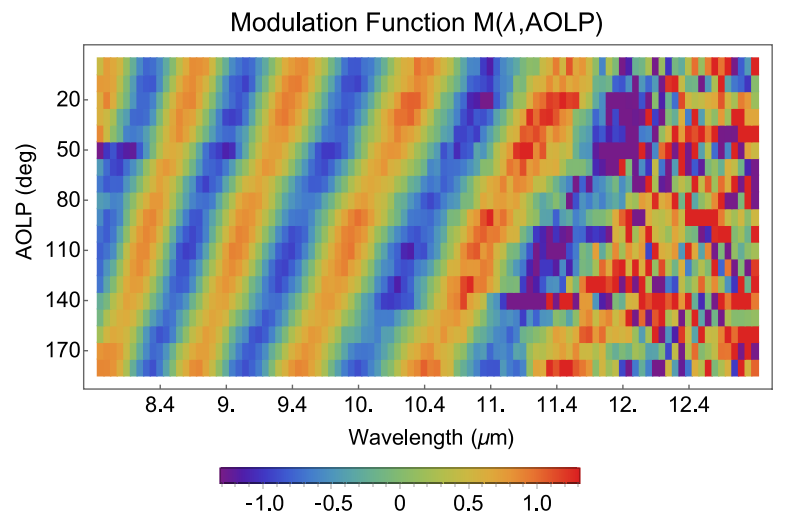

Fig. 10 The modulation function $\hat{M}$ [Eq. (9)] computed from fully polarized narrowband calibration measurements as a function of AOLP and wavelength. The modulation function is degraded at wavelengths higher than $11 \mu \mathrm{m}$ due to the camera SNR reported in Fig. 9.

\subsubsection{Fit to modulation function}

To calculate the modulation function, first the intensity profiles of the signals in paths 1 and 2 are corrected for transmission using the unpolarized calibration measurements in Fig. 6. Then, the modulation function for each input AOLP is calculated using Eq. (9). As expected, the phase of the measured modulation function will shift with AOLP as seen in Fig. 10. At longer wavelengths, the modulation function loses its expected appearance as increased noise in both cameras is compounded by applying Eq. (9). Figure 11 shows the fit of the measured modulation as a

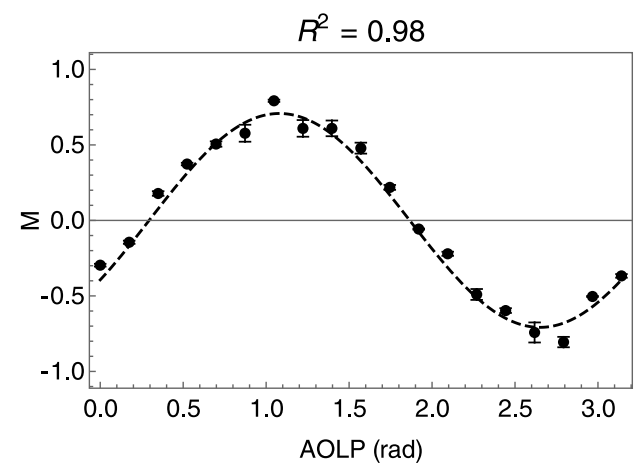

(a) $\lambda=8.5 \mu m$

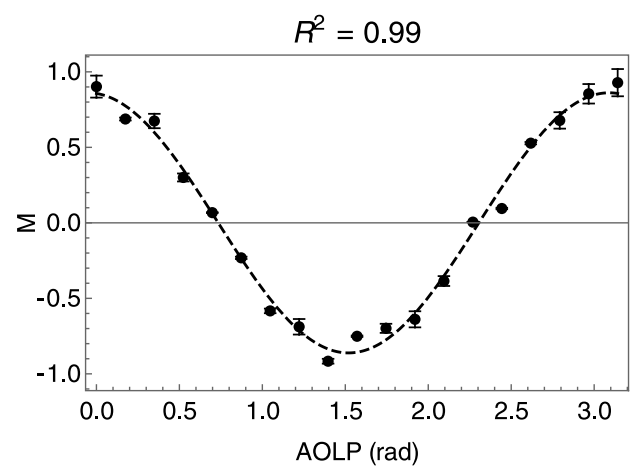

(c) $\lambda=10.5 \mu m$

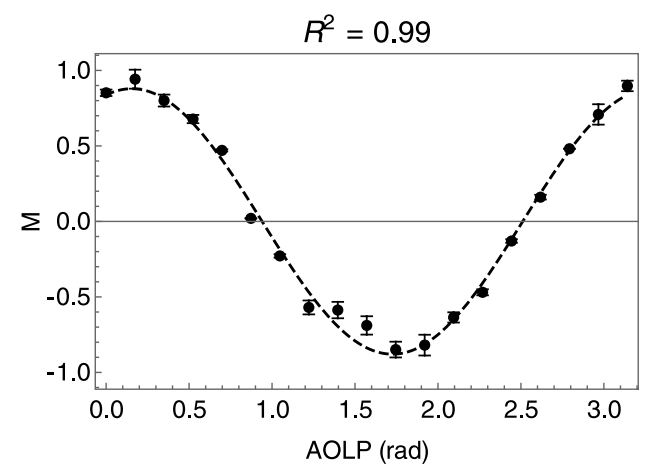

(b) $\lambda=9.5 \mu m$

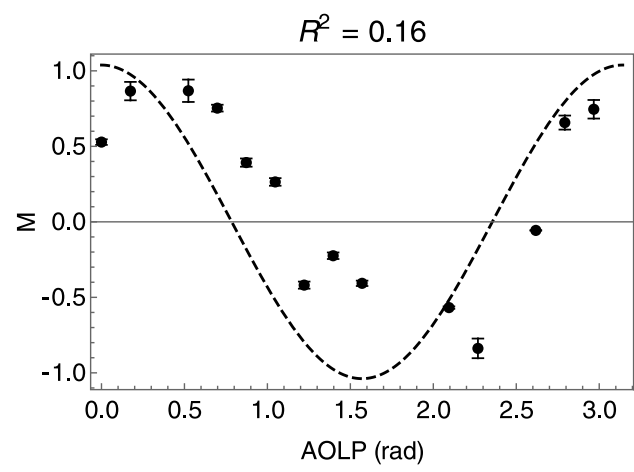

(d) $\lambda=11.5 \mu \mathrm{m}$

Fig. 11 Fit of measured modulation function to Eq. (9) for $\lambda=8.5,9.5,10.5$, and $11.5 \mu \mathrm{m}$. $R^{2}$ values [Eq. (12)] for each fit are shown above figures. As with the Mueller matrix model fits (Fig. 7), there is a significant reduction in $R^{2}$ past $11 \mu \mathrm{m}$. 


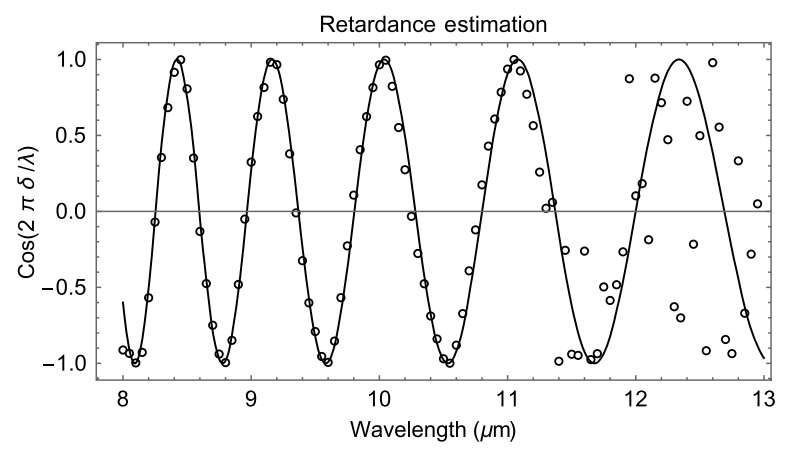

Fig. 12 Estimated retardance from calibration data. Solid line is the calculated retardance for the CdSe dispersion model with a thickness of $5.01 \mathrm{~mm}$. Dots are retardance estimated at each wavelength during narrowband calibration. Agreement between the two at shorter wavelengths indicates that the polarization optics are performing as intended. The abrupt deviation from the expected retardance modulation above $11 \mu \mathrm{m}$ will reduce the accuracy of polarimetric retrievals at these longer wavelengths.

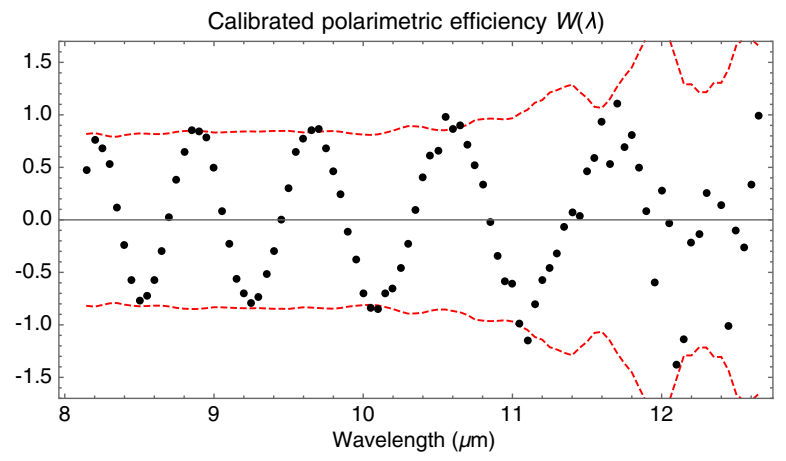

Fig. 13 Polarimetric efficiency of the system $\hat{W}(\lambda)$ for narrowband light in red plotted over the measured modulation for $\mathrm{AOLP}=0$. Efficiency is greater than 0.8 from 8 to $11 \mu \mathrm{m}$.

function of AOLP to Eq. (9). The result of this fit and the associated $R^{2}$ values are shown for 8.5, 9.5, 10.5, and $11.5 \mu \mathrm{m}$.

When fitting to the modulation function in Eq. (9), both the polarimetric efficiency $\hat{W}$ and retardance $\hat{\delta}$ are estimated as for each measured wavelength. Figure 12 shows the retardance calculated using a known dispersion model for CdSe as well as the retardance estimated by fitting the measured data to Eq. (9) at each wavelength during calibration. From 8 to $11 \mu \mathrm{m}$ the estimated retardance shows good agreement with the expected result. Figure 13 shows the estimated efficiency $\hat{W}$ plotted over the measured modulation for AOLP $=0$. In the ideal system, the modulation function should range from -1 to 1 with an amplitude of 2 . For the narrowband monochromator data, $\hat{W}$ ranges from 0.8 to 0.9 from 8 to $11 \mu \mathrm{m}$. Since the spectral blurring is minimized for this data, the difference in contrast in reflection and transmission is the main source of reduction of the polarimetric efficiency $\hat{W}$ for the narrowband case. The accuracy of polarimetric retrievals and this efficiency are highly dependent, ${ }^{16}$ and thus the performance of the IRCSP could be improved by a higher contrast polarizing beam splitter such as a Wollaston prism.

For the monochromator data, we expect the response to be well characterized by polarimetric behavior alone, as spectral blurring is minimized. Figure 14 compared the $R^{2}$ values for both the Mueller matrix and modulation models as a function of measured wavelengths. At all wavelengths, the Mueller matrix model outperforms the modulation function, with this difference becoming compounded past $11 \mu \mathrm{m}$. 


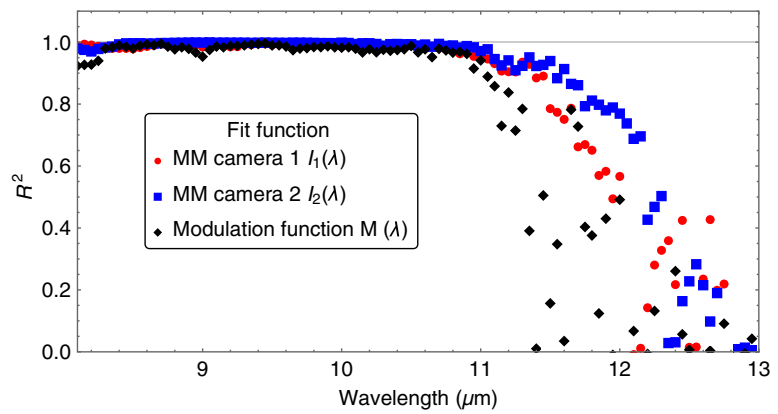

Fig. 14 Comparison of the coefficient of determination $\left(R^{2}\right)$ for the fit of the calibration data to the Mueller matrix model for $I_{c}(\lambda)$ and the modulation function $M(\lambda)$.

\section{Conclusion}

This project has demonstrated the feasibility of uncooled, low-cost, compact LWIR polarimeters using currently existing thermal imaging technology. This first light demonstration of the instrument confirms the anticipated response to polarized light and agreement with the Mueller matrix model. Once fully calibrated in an environmental chamber, the current IRCSP will be tested in the field to perform snapshot thermal spectropolarimetric measurements. Deployed, this instrument will produce the first spectropolarimetric measurements of optically thin cirrus ice clouds in the thermal IR.

The analysis presented here demonstrated that a Mueller matrix model is effective at describing the IRCSP performance for narrowband calibration measurements. The measured efficiency exceeds 0.8 from 8 to $11 \mu \mathrm{m}$ indicating that polarimetric retrievals are plausible using this design. For narrowband light, the performance is most limited by the reduction in SNR at longer wavelengths for the uncooled-microbolometer. Polarimetric efficiency will be reduced for broadband measurements, due to spectral blurring. This will further reduce polarimetric accuracy for longer wavelengths. The selection of a narrower field stop is a design parameter which can be tuned to reduce spectral blurring. ${ }^{31}$

While this prototype successfully demonstrates the instrument concept, improvements in the SNR of the instrument must be made before the instrument can be qualified for spaceflight and be suitable for cold target measurement. Future studies utilizing this prototype IRCSP will enable quantification of minimum SNR requirements for desired accuracy in AOLP and DOLP measurements. ${ }^{31}$ An SNR performance study is needed to inform the cost-benefit of acquiring custom optics and detectors for a space qualified system. The temperature reduction in deployment $\left(-5^{\circ} \mathrm{C}\right)$ could provide an SNR improvement which yields accurate polarimetric measurements of cold targets. Environmental chamber tests will determine how the instrument SNR varies and improves as the ambient temperature is lowered. As passive cooling is possible for the IRCSP during spaceflight or high-altitude deployment, it is plausible that the reduction of thermal background and detector noise from lower ambient temperatures will be sufficient. As microbolometer technology continues to improve and more compact detectors become available, it may be possible in the near future to replace the FLIR Boson with a more sensitive detector.

While designed for cloud ice measurement, the IRCSP's form factor and cost make it a candidate for a variety of future thermal polarimetric sensing applications including ocean surface normal estimation, atmospheric monitoring of aerosols, and the study of thermal polarimetric behavior as a function of wavelength and target temperature. This first light demonstration motivates the demand for optical components not readily available in the LWIR such as polarization gratings and Wollaston prisms, in addition to an increased selection of lenses, gratings, and waveplates. These advances will support the innovation of easily deployable uncooled LWIR polarimeters for a wide range of applications made feasible by modern microbolometers.

\section{Acknowledgments}

The authors acknowledge the NASA Earth, Space, and Technology Office for funding this work. The authors would like to thank collaborators at both NASA Goddard Spaceflight Center and the 
University of Arizona for their contributions: Chang Jin Oh and the Large Optics Testing and Fabrication facility for their assistance with the optomechanical design; Giovanni De Amici, Aaron Pearlman, Peter Pantina, and Manuel Vega at GSFC for project support; Todd Horne for his assistance with instrument assembly; Jeremy Parkinson and Kyler Langworthy for data collection; and Adriana Stohn for her work on the Boson data acquisition software. Disclosures: The authors have no relevant financial interests in the manuscript and no other potential conflicts of interest to disclose.

\section{References}

1. ESTO, ESTO 2016 Report, NASA, https://esto.nasa.gov/wp-content/uploads/2020/03/ Microwave_TechStrategy_2016.pdf (2016).

2. D. L. Wu et al., "Toward global harmonization of derived cloud products," Bull. Am. Meteorol. Soc. 98(2), ES49-ES52 (2017).

3. D. L. Wu et al., "Comparisons of global cloud ice from MLS, CloudSat, and correlative data sets," J. Geophys. Res. 114, D00A24 (2009).

4. G. Liu, "A database of microwave single-scattering properties for nonspherical ice particles," Bull. Am. Meteorol. Soc. 89(10), 1563-1570 (2008).

5. S. S. Board et al., Earth Science and Applications from Space: National Imperatives for the Next Decade and Beyond, National Academies Press, Washington, DC (2017).

6. X. Xie and J. Miao, "Polarization difference due to nonrandomly oriented ice particles at millimeter/submillimeter waveband," J. Quant. Spectrosc. Radiat. Transfer 112(6), 10901098 (2011).

7. L. Bi and P. Yang, "Accurate simulation of the optical properties of atmospheric ice crystals with the invariant imbedding T-matrix method," J. Quant. Spectrosc. Radiat. Transfer 138, 17-35 (2014).

8. P. Yang et al., "Spectrally consistent scattering, absorption, and polarization properties of atmospheric ice crystals at wavelengths from 0.2 to $100 \mu \mathrm{m}$," J. Atmos. Sci. 70(1), 330-347 (2013).

9. K. Liou and P. Yang, Light Scattering by Ice Crystals, Cambridge University Press, Cambridge, United Kingdom (2016).

10. J. Miao et al., "The potential of polarization measurements from space at $\mathrm{mm}$ and sub-mm wavelengths for determining cirrus cloud parameters," Atmos. Chem. Phys. 3(1), 39-48 (2003).

11. J. Gong and D. L. Wu, "Microphysical properties of frozen particles inferred from global precipitation measurement (GPM) microwave imager (GMI) polarimetric measurements," Atmos. Chem. Phys. 17(4), 2741-2757 (2017).

12. J. J. Coy et al., "Sensitivity analyses for the retrievals of ice cloud properties from radiometric and polarimetric measurements in sub- $\mathrm{mm} / \mathrm{mm}$ and infrared bands," J. Geophys. Res.: Atmos. 125, e2019JD031422 (2020).

13. B. A. Baum et al., "Ice cloud single-scattering property models with the full phase matrix at wavelengths from 0.2 to $100 \mu \mathrm{m}$," J. Quant. Spectrosc. Radiat. Transfer 146, 123-139 (2014).

14. K. A. Hart, R. A. Chipman, and D. L. Wu, "Compact LWIR polarimeter for cirrus ice properties," Proc. SPIE 10655, 106550V (2018).

15. K. Oka and T. Kato, "Spectroscopic polarimetry with a channeled spectrum," Opt. Lett. 24(21), 1475-1477 (1999).

16. J. M. Smit et al., "SPEX airborne spectropolarimeter calibration and performance," Appl. Opt. 58(21), 5695-5719 (2019).

17. J. Rietjens et al., "SPEX: a highly accurate spectropolarimeter for atmospheric aerosol characterization," Proc. SPIE 10563, 1056344 (2017).

18. J. H. Rietjens et al., "Accurate spectrally modulating polarimeters for atmospheric aerosol characterization," Proc. SPIE 9613, 96130S (2015).

19. F. J. Iannarilli et al., "Snapshot LWIR hyperspectral polarimetric imager for ocean surface sensing," Proc. SPIE 4133, 270-284 (2000). 
20. F. J. Iannarilli et al., "Polarimetric-spectral intensity modulation (P-SIM): enabling simultaneous hyperspectral and polarimetric imaging," Proc. SPIE 3698, 474-482 (1999).

21. J. Craven-Jones et al., "Thermally stable imaging channeled spectropolarimetry," Proc. SPIE 8873, 88730J (2013).

22. F. Snik et al., "SPEX: the spectropolarimeter for planetary exploration," Proc. SPIE 7731, 77311B (2010).

23. D. J. Diner et al., "Dual-photoelastic-modulator-based polarimetric imaging concept for aerosol remote sensing," Appl. Opt. 46(35), 8428-8445 (2007).

24. H. E. Scott, S. H. Jones, and F. J. Iannarilli, "Imaging infrared polarimetry: initial results and potential in detection of scatterable mines and surface disturbances," Proc. SPIE 3710, 1188-1212 (1999).

25. G. van Harten et al., "Spectral line polarimetry with a channeled polarimeter," Appl. Opt. 53(19), 4187-4194 (2014).

26. F. Snik, T. Karalidi, and C. U. Keller, "Spectral modulation for full linear polarimetry," Appl. Opt. 48(7), 1337-1346 (2009).

27. P. W. Nugent and J. A. Shaw, "Calibration of uncooled LWIR microbolometer imagers to enable long-term field deployment," Proc. SPIE 9071, 90710V (2014).

28. D. Chenault et al., "Pyxis: enhanced thermal imaging with a division of focal plane polarimeter," Technical Report, Polaris Sensor Technologies, Inc. (2015).

29. D. B. Chenault et al., "New IR polarimeter for improved detection of oil on water," SPIE Newsroom (2018).

30. R. A. Chipman, T. Lam, and G. Young, Polarized Light and Optical Systems, 1st ed., CRC Press, New York (2019).

31. K. A. Hart et al., "Linear Stokes measurement of thermal targets using compact LWIR spectropolarimeter," Proc. SPIE 11412, 91-103 (2020).

32. Airy Optics, "Polaris-M polarization ray tracing software," 2019, http://www.airyoptics .com/polaris-m-software/.

33. M. Lisitsa et al., "Dispersion of the refractive indices and birefringence of cdsxse1- $\mathrm{x}$ single crystals," Phys. Status Solidi (B) 31(1), 389-399 (1969).

34. D. B. Chenault and R. A. Chipman, "Infrared birefringence spectra for cadmium sulfide and cadmium selenide," Appl. Opt. 32, 4223-4227 (1993).

35. P. D. Hale and G. W. Day, "Stability of birefringent linear retarders (waveplates)," Appl. Opt. 27, 5146-5153 (1988).

36. Laboratory of Physical-Chemical Basis of Crystallization (LPCBC), Cadmium Selenide (CdSe) Datasheet (2002).

37. P. Wu et al., "Growth and characterization of zinc and cadmium thiogallate," Mater. Res. Bull. 23(11), 1605-1609 (1988).

Kira A. Hart is a PhD candidate in the Wyant College of Optical Science at the University of Arizona. She received her BS degree in physics from the UCLA Honor's College in 2019. Currently, she is a member of the Polarization Lab under the advisement of Dr. Russell Chipman where her research is focused on the design of LWIR polarimeters for remote sensing and target detection applications. She is a member of SPIE.

Meredith Kupinski is a research professor of the College of Optical Sciences at the University of Arizona. She received her MS and PhD degrees in optical science from the University of Arizona in 2003 and 2008, respectively. Her research interests include task-relevant metrics for imaging system design, estimation/detection theory, and stochastic systems analysis and information quantitation. She received NSF's fellowship for science, engineering, and education for sustainability in 2013 to 2016.

Dong Wu is a NASA scientist at Goddard Space Flight Center (GSFC). He is the project scientist of NASA's Solar Radiation and Climate Experiment (SORCE) and Total and Spectral Solar Irradiance Sensor (TSIS) missions. His research is focused on remote sensing of clouds from space and better understanding of sun-climate connection, primarily in $\mathrm{mm}$ - and submmwave remote sensing of ice clouds, stereo imaging for cloud-track winds, and precision 
radiometry for atmospheric gravity waves. Other research includes radio occultation techniques for E-region electron density, and $2 \mathrm{THz}$ techniques for thermospheric winds and oxygen density.

Russell A. Chipman is a professor of optical sciences at the University of Arizona and a visiting professor at the Center for Optics Research and Education, Utsunomiya University, Japan. He received his BS degree in physics from MIT and his MS and PhD degrees in optical science from the University of Arizona. He is a fellow of OSA and SPIE and a coauthor of the textbook Polarized Light and Optical Systems. He received SPIE's 2007 G. G. Stokes award for research in polarimetry and OSA's Joseph Fraunhofer award/Robert Burley award for optical engineering in 2015 . 